\title{
Extensive multiband study of the X-ray rich GRB 050408 ^
}

\section{A likely off-axis event with an intense energy injection $\star \star$}

A. de Ugarte Postigo ${ }^{1}$, T. A. Fatkhullin ${ }^{2}$, G. Jóhannesson ${ }^{3}$, J. Gorosabel ${ }^{1}$, V. V. Sokolov ${ }^{2}$, A. J. Castro-Tirado ${ }^{1}$, Yu. Yu. Balega ${ }^{2}$, O. I. Spiridonova ${ }^{2}$, M. Jelínek ${ }^{1}$, S. Guziy ${ }^{1,4}$, D. Pérez-Ramírez ${ }^{5}$, J. Hjorth ${ }^{6}$, P. Laursen ${ }^{6}$, D. Bersier ${ }^{7}$,

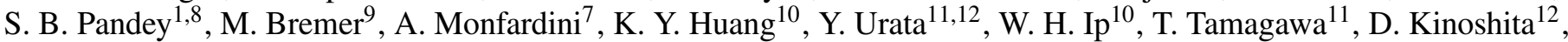
T. Mizuno ${ }^{13}$, Y. Arai ${ }^{13}$, H. Yamagishi ${ }^{13}$, T. Soyano ${ }^{14}$, F. Usui ${ }^{15}$, M. Tashiro ${ }^{16}$, K. Abe ${ }^{16}$, K. Onda ${ }^{16}$, Z. Aslan ${ }^{17,18}$, I. Khamitov ${ }^{17}$, T. Ozisik ${ }^{17}$, U. Kiziloglu ${ }^{19}$, I. Bikmaev ${ }^{20,21}$, N. Sakhibullinn ${ }^{20,21}$, R. Burenin ${ }^{22}$, M. Pavlinsky ${ }^{22}$, R. Sunyaev ${ }^{22}$, D. Bhattacharya ${ }^{23}$, A. P. Kamble ${ }^{23}$, C. H. Ishwara Chandra ${ }^{24}$, and S. A. Trushkin ${ }^{2}$

1 Instituto de Astrofísica de Andalucía (IAA-CSIC), Apartado de Correos 3004, 18080 Granada, Spain e-mail: deugarte@iaa.es

2 Special Astrophysical Observatory, Nizhnij Arkhyz, Zelenchokskaya, Karachaevo-Cherkesia, 369167 Russia

3 Science Institute, University of Iceland, Dunhaga 3, 107 Reykjavík, Iceland

4 Nikolaev State University, Nikolska 24, 54030, Nikolaev, Ukraine

Dpto. de Física (EPS), Universidad de Jaén, 23071 Jaén, Spain

Dark Cosmology Centre, Niels Bohr Institute, University of Copenhagen, Juliane Maries Vej 30, 2100 Copenhagen, Denmark

Astrophysics Research Inst., Liverpool John Moores Univ., Twelve Quays House, Egerton Wharf, Birkenhead, CH41 1LD, UK

The UCL Mullard Space Science Laboratory, Holmbury St. Mary, Dorking, Surrey, RH5 6NT, UK

9 Institut de Radio Astronomie Millimétrique (IRAM), 300 rue de la Piscine, 38406 Saint-Martin d'Hères, France

10 Institute of Astronomy, National Central University, Chung-Li 32054, Taiwan, PR China

11 Institute for Physics and Chemical Research (RIKEN), Wako, Saitama 351-0198, Japan

12 Tokyo Institute of Technology, Ookayama, Meguro, Tokyo 152-8550, Japan

13 Department of Astronomy and Earth Sciences, Tokyo Gakugei University, Koganei, Tokyo 184, Japan

14 Kiso Observatory, Institute of Astronomy, University of Tokyo, Mitake-mura, Kiso-gun, Nagano 397-0101, Japan

15 Japan Aerospace Exploration Agency, Institute of Space and Astronautical Science, Sagamihara, Kanagawa 229-8510, Japan

16 Saitama University, Sakura-ku, Saitama 338-8570, Japan

17 TÜBITAK National Observatory, Akdeniz Üniversitesi, 07058, Antalya, Turkey

18 Akdeniz University, Physics Department, 07058 Antalya, Turkey

19 Middle East Technical University, Physics Department, Inonu Bulvari, Ankara, 06531, Turkey

20 Departments of Astronomy, Kazan State University, Kremlevskaya Str., 18, Kazan, 420008, Russia

21 Academy of Sciences of Tatarstan, Bauman Str., 20, Kazan, 420111, Russia

22 Space Research Institute (IKI), 84/32 Profsoyuznaya, Moscow, 117997, Russia

23 Raman Research Institute, Bangalore 560 080, India

24 National Centre for Radio Astrophysics, Ganeshkhind, Pune, 411007, India

Received 30 October 2006 / Accepted 14 December 2006

\section{ABSTRACT}

Aims. Understand the shape and implications of the multiband ligth curve of GRB 050408, an X-ray rich (XRR) burst. Methods. We present a multiband optical light curve, covering the time from the onset of the $\gamma$-ray event to several months after, when we only detect the host galaxy. Together with X-ray, millimetre and radio observations we compile what, to our knowledge, is the most complete multiband coverage of an XRR burst afterglow to date.

Results. The optical and X-ray light curve is characterised by an early flattening and an intense bump peaking around 6 days after the burst onset. We explain the former by an off-axis viewed jet, in agreement with the predictions made for XRR by some models, and the latter with an energy injection equivalent in intensity to the initial shock. The analysis of the spectral flux distribution reveals an extinction compatible with a low chemical enrichment surrounding the burst. Together with the detection of an underlying starburst host galaxy we can strengthen the link between XRR and classical long-duration bursts.

Key words. gamma rays: bursts - techniques: photometric

^ Based on observations collected at SAO, La Silla, Roque de los Muchachos, Haleakala, Kitt Peak, Cerro Tololo, TÜBITAK, Kiso, Observatorio de Sierra Nevada, Plateau du Bure, GMRT and RATAN-600.

$\star \star$ Appendices A and B are only available in electronic form at http://www . aanda.org

\section{Introduction}

X-ray flashes (XRFs) where first identified by Beppo-SAX (Heise et al. 2001) as those bursts detected by the X-ray camera but not the $\gamma$-ray monitor. Later studies based on the larger sample gathered by HETE-2 (Sakamoto et al. 2005b) gave a more 
general (and instrument- independent) classification and confirmed the intermediate group of events, the X-ray rich (XRR) class, previously detected by Ginga (Yoshida \& Murakami 1994) and Granat/WATCH (Castro-Tirado et al. 1994). It is now known that long-duration GRBs (LGRBs), XRRs and XRFs share the same isotropic distribution in the sky, the same duration range and similar spectrum, with the main difference of having respectively lower observed spectral peak energy $E_{\text {peak }}^{\text {obs }}$ in the $v F_{v}$ spectrum. They seem to form a continuum and thereby, most of the proposed models have tried to explain them as a unified phenomena (see a summary of the different models in Granot et al. 2005).

GRB 050408 was detected by WXM, SXC, and FREGATE aboard HETE-2 (Sakamoto et al. 2005a) at 2005 April 08 16:22:50.93 UT ( $t_{0}$ hereafter). With an observed peak energy of $\sim 20 \mathrm{keV}$ it was classified as an XRR event. The $1.0 \mathrm{~m}$ Zeiss and $6.0 \mathrm{~m}$ BTA telescopes at the Special Astrophysical Observatory (SAO) in Russia pointed at the position delivered by HETE-2 through the GCN (GRB Circular Network) and detected the optical afterglow (de Ugarte Postigo et al. 2005a) coincident with the possition of the X-ray afterglow detected by Swift/XRT, which began observing 42 min after the burst (Wells et al. 2005). The precise localisation allowed further optical (Covino et al. 2007) and spectroscopic (Berger et al. 2005; Prochaska et al. 2005) observations, this latter ones detemined a redshift of $z=1.236$.

In Sect. 2 we present the observations and the reduction methods that have been used for the analysis of the data. Section 3 describes the results that have been obtained, including observations of the host galaxy and modelling of the light curve. Section 4 discusses the implications of the analysis of the light curve.

\section{Observations and data reduction}

For this work we have compiled over 60 photometric measurements in $U, B, V, R \mathrm{c}$ and $I \mathrm{c}$ bands from 12 telescopes. The images where reduced using standard techniques based on IRAF ${ }^{1}$ and JIBARO (de Ugarte Postigo et al. 2005b).

The burst happened during night time in Japan, where a fast follow up was carried out. The very wide field camera, WIDGET was monitoring the field of view of HETE-2 when the event was reported but detected no optical emission before, during or after the gamma-ray emission down to an unfiltered limiting magnitude of 9.7 (all limits given throughout the paper are $3-\sigma)$. The $1.05 \mathrm{~m}$ KISO Schmidt telescope pointed to the error box 20 min after the burst but failed to detect the afterglow. Finally, the $1 \mathrm{~m}$ LOT telescope observed the field $55 \mathrm{~min}$ after the burst, images that later served to confirm the afterglow (Huang et al. 2005b). The discovery of the afterglow was made with the data of the $1.0 \mathrm{~m}$ Zeiss and $6.0 \mathrm{~m}$ BTA telescopes in Russia (de Ugarte Postigo et al. 2005a), starting 115 min after the burst, when observations became possible from that site.

Further observations were performed from the $1.5 \mathrm{~m}$ Russian-Turkish telescope, in TÜBITAK National Observatory, the $4.0 \mathrm{~m}$ Blanco telescope in Cerro Tololo, the $4.0 \mathrm{~m}$ Mayall telescope in Kitt Peak, the $2.0 \mathrm{~m}$ Faulkes Telescope North (FTN) in Haleakala and the $3.5 \mathrm{~m}$ Telescopio Nazionale Galileo (TNG) in la Palma. A specially intense multiband campaign was carried out from the $1.54 \mathrm{~m}$ Danish telescope in La Silla, where daily

1 IRAF is distributed by the National Optical Astronomy Observatories, which is operated by the Association of Universities for Research in Astronomy, Inc. (AURA) under cooperative agreement with the National Science Foundation. observations were obtained during the first 8 days following the burst onset.

Finally, 8 months after the burst, deep observations were made from the $3.5 \mathrm{~m}$ telescope at Calar Alto. In these images we detect the host galaxy of the burst in $B$ and $R \mathrm{c}$ bands and impose a limit in Ic band.

Optical photometric calibration is based on the observation of several standard fields (Landolt 1992) using the $1.54 \mathrm{~m}$ Danish telescope at La Silla and the $1.5 \mathrm{~m}$ telescope at Sierra Nevada Observatory. From these observations we derive $12 \mathrm{sec}-$ ondary standards of different brightnesses. A log with the observations and the calibration stars are given as online material.

Our dataset is completed with several millimetre and radio limits. 6 epochs of millimetre observations were carried out with the 6-antenna Plateau de Bure interferometer (PdB, Guilloteau et al. 1992). No detection was obtained in either of the $1 \mathrm{~mm}$ or $3 \mathrm{~mm}$ bands, although a $3-\sigma$ signal was found on the phase center in both observing bands on April 18. Careful re-analysis of the data did not reveal these signals as instrumental artifacts. Based on the extreme spectral slope and the non-detection on April 19, we conclude that this result is either due to a statistical fluctuation or an unusual event of interstellar scattering at high galactic latitude, and not due to a source-intrinsic variation. Data calibration was done using the GILDAS software package ${ }^{2}$ using MWC349 as primary flux calibrator and 3C 273 as amplitude and phase calibrator. Further observations where obtained 13 days after the burst at $1.28 \mathrm{GHz}$ from GMRT and at $8.4 \mathrm{GHz}$ from RATAN-600.

\section{Results}

\subsection{Light curve}

In order to put together all the radio, millimetre, near infrared (nIR), optical and X-ray data available (including data from Foley et al. 2006 and Soderberg 2005a,b), we have determined the corresponding flux density values for all observations. X-ray afterglow counts, obtained from Foley et al. (2006) have been converted and corrected for hydrogen column extinction using WEBPIMMS ${ }^{3}$ taking as spectral model a powerlaw with a slope of $\beta_{\mathrm{X}}=1.16$ and a column density of $N_{\mathrm{H}}=0.25 \times$ $10^{22} \mathrm{~cm}^{-1}$ (Nousek et al. 2006; Chincarini et al. 2005). The optical data have been corrected for galactic reddening (using an $E(B-V)=0.026$, Schlegel et al. 1998) and intrinsic extinction (see Sect. 3.2). The measured/estimated host galaxy flux has been subtracted from the data to obtain the clean afterglow flux (see Sect. 3.3). The conversion of the optical data to flux density was done using the transformations given by Fukugita et al. (1995) for the optical and by Allen et al. (2000) for the nIR. The resulting light curves are shown in Fig. 1. Note the intense bump, rising at $\sim 3$ days and peaking at $\sim 6$ days, both in optical and X-rays. These kind of fluctuations have already been detected in the light curves of LGRBs and short-duration bursts (SGRBs) (de Ugarte Postigo et al. 2005c, 2006).

\subsection{Study of the optical-nIR SFD}

We have constructed the UBVRcIcJHK-band Spectral Flux distribution (SFD) of the afterglow 0.6 days after the burst, when near simultaneous optical and nIR observations were available.

\footnotetext{
${ }^{2}$ GILDAS is the software package distributed by the IRAM Grenoble GILDAS group.

${ }^{3}$ http://heasarc.nasa.gov/Tools/w3pimms.html
} 


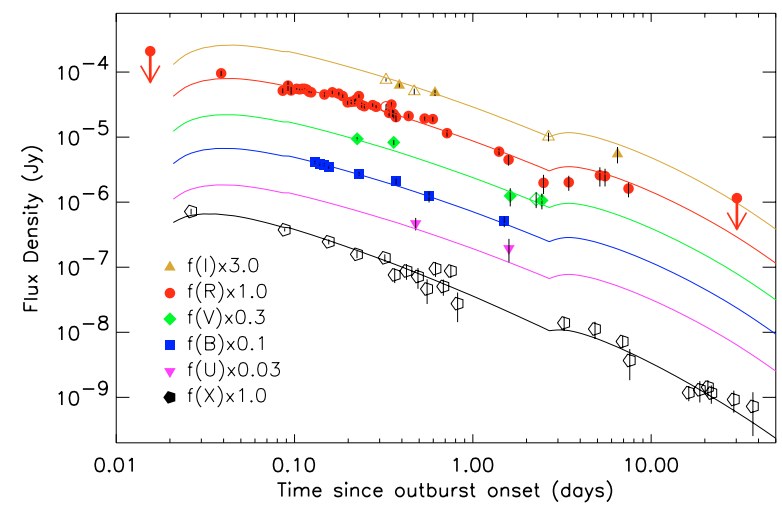

Fig. 1. Multiwavelength light curve of the GRB 050408 afterglow in the observer frame. The lines show the best fit of a fireball model with one energy injection (at 2.9 days) seen off-axis (see text for details). Our observations are plotted with filled symbols, while the ones obtained from the literature are represented by empty ones, this convention is used for all the figures.

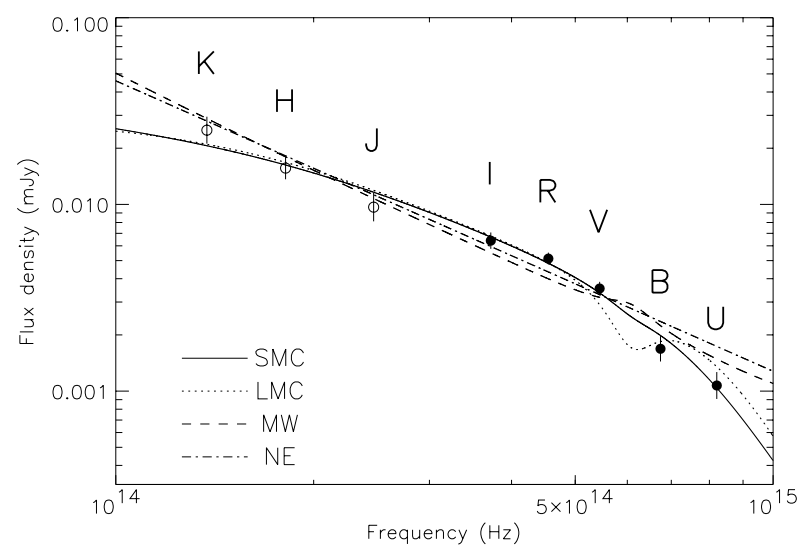

Fig. 2. Spectral flux distribution of the afterglow 0.6 days after the burst onset in the observer frame. The different lines represent results from fitting the data to various extinction laws: Small Magellanic Cloud (SMC), Large Magellanic Cloud (LMC), Milky Way (MW) and No Extinction (NE).

The UBVRcIc-band magnitudes from this work were complemented with the $J H K$-band values reported by Foley et al. (2006). Synchronisation to a unique timing is done by assuming a powerlaw with an index of $\alpha=0.7\left(F \propto t^{-\alpha}\right)$, as derived from a linear fit of the nearby multiband data of the afterglow.

The fluxes are used for fitting an extincted powerlaw $\left(F_{v} \propto\right.$ $10^{-0.4 A_{v}} v^{-\beta}$ ) with 3 different extinction laws: Milky Way (MW), Large Magellanic Cloud (LMC) and Small Magellanic Cloud (SMC) as described by Pei (1992). This allows us to obtain $A_{\mathrm{V}}$ and $\beta$ simultaneously. The results of these 3 fits are complemented with an unextincted powerlaw case (NE), see Fig. 2 and Table 1. The best fit to the SFD of the afterglow is obtained when considering a SMC extinction law $\left(\chi^{2} /\right.$ d.o.f. $\left.=5.0 / 5\right)$. This is consistent with what has been previously found for other LGRB afterglows (Kann et al. 2006).

\subsection{The host galaxy}

Several months after the gamma-ray event we revisited the GRB field with the $3.5 \mathrm{~m}$ telescope at Calar Alto Observatory in order to search for the host galaxy. Images were obtained in $B, R \mathrm{c}$ and $I \mathrm{c}$ bands, yielding a faint detection in $B$ and $R \mathrm{c}$
Table 1. Results of the SFD fitting at 0.6 days for different extinction laws.

\begin{tabular}{cccc}
\hline \hline Extinction law & $\beta$ & $A_{\mathrm{V}}$ & $\chi^{2} /$ d.o.f. \\
\hline MW & $1.85 \pm 0.30$ & $-0.18 \pm 0.22$ & $24.5 / 5$ \\
LMC & $-0.12 \pm 0.48$ & $1.19 \pm 0.32$ & $11.5 / 5$ \\
SMC & $0.28 \pm 0.33$ & $0.73 \pm 0.18$ & $5.0 / 5$ \\
NE & $1.62 \pm 0.07$ & 0 & $21.0 / 5$ \\
\hline
\end{tabular}

and imposing a limit on Ic. We derive galaxy colour indices of $(B-R \mathrm{c})=0.7 \pm 0.5$ and $(R \mathrm{c}-I \mathrm{c}) \leq 0.73$. These values are corrected for Galactic extinction. We have compared these values to the ones derived from the templates computed by Kinney et al. (1996) for a wide variety of galaxy types. We may conclude that only starburst galaxy templates are consistent with them. The best correlation is obtained with the starbust 2 template, with an intrinsic extinction of $E(B-V)=0.16$.

\subsection{Modelling of the multiband data}

Using the model and methods described by Jóhannesson et al. (2006) we fitted the multiband observations of the afterglow (galaxy subtracted) to a fireball model with energy injections, viewed both on-axis and off-axis (with varying viewing angles). At least one injection is needed in order to account for the bump seen at 6 days which would carry as much energy as the initial shock. Another characteristic of the light curve is a flattening of the early light curve, seen in Rc and X-rays during the first hours of the burst, which has already been reported by Foley et al. (2006). This can be explained either by an early energy injection (single or continous), or an outflow with a low initial Lorentz factor, or as the result of an off-axis viewed burst. Similar early behaviour has already been found in other bursts (Nousek et al. 2006; Zhang et al. 2006).

Our preferred scenario (giving the best fit) describes the burst as a collimated $\left(\theta_{0}=2.7^{\circ}\right)$ fireball seen off axis $\left(\theta_{v}=\right.$ $\left.1.45 \theta_{0}\right)$ expanding into a uniform low density environment $\left(n_{0}=\right.$ $0.01 \mathrm{~cm}^{-3}$ ) with an electron index $p=2.03$ and having an additional energy injection after 2.9 days with 1.2 times the initial energy. No further injections are needed to explain the light curve with the available amount of data. From the fit we obtain a $\chi^{2} /$ d.o.f. $=158.1 / 93$. The jet break, as defined by Sari et al. (1999), would be expected initially at 1.6 days, or at 3.8 days due to the energy injection. However, due to the effect of the equal arrival time surface, it is further delayed to approximatelly 30 days. Figure 3 shows the radio to X-ray SFD predicted by our model for 3 epochs, together with observational data, host subtraced and corrected for galactic and intrinsic extinction.

\section{Discussion}

The optical-nIR SFD shows a clear curvature, implying a need for extinction along the afterglow line of sight in the host galaxy. The only reliable fit $(\chi /$ d.o.f. $=5.0 / 5)$ is based on a SMC extinction law. This result points towards a low stage of chemical enrichment in the region of the progenitor, as is usually found for LGRBs (Kann et al. 2006). The detection of a starburst host galaxy is also a common feature with most LGRBs (Fruchter et al. 2006), facts that once again favour the hypothesis of shared nature between XRR bursts and LGRBs.

The optical spectral index obtained from this fit $\left(\beta_{\mathrm{o}}=0.28 \pm\right.$ $0.33)$ and the $\mathrm{X}$-ray one $\left(\beta_{\mathrm{X}}=1.14 \pm 0.19\right.$, Nousek et al. 2006) are consistent with a synchrotron spectra in which the cooling 


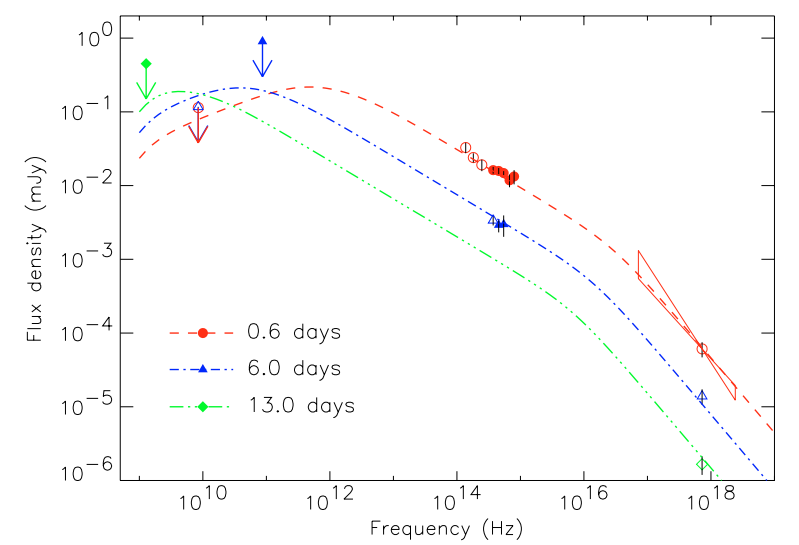

Fig. 3. Spectral Flux Distribution of the afterglow from radio to X-rays $0.6,6.0$ and 13.0 days after the burst in the observer frame. Several $3-\sigma$ upper limits from radio and millimetre observations are plotted.

break frequency $\left(v_{\mathrm{c}}\right)$ is located between optical and X-rays. A linear fit of the optical $(R \mathrm{c})$ and $\mathrm{X}$-ray data between 0.1 and 1.0 days (where there is more data available and the light curve seems more stable) returns temporal slope values of $\alpha_{\mathrm{o}}=0.69 \pm$ 0.04 and $\alpha_{\mathrm{X}}=0.99 \pm 0.21$. These numbers, together with the optical and X-ray spectral slopes, are consistent with a standard fireball model (Sari et al. 1999) in which a relativistic outflow is expanding in a uniform density environment in the slow cooling regime with an electron power law distribution index of $p \sim 2.0$.

A more complex multirange model, confirms these results and is used to account for the bump that has been detected to peak at $\sim 6$ days by allowing for refreshed shocks. This fluctuation is simultaneously observed in optical and X-rays and can be explained by an energy injection of the order of the initial shock. This achromaticity and the simultaneity at both sides of $v_{\mathrm{c}}$ rules out other explanations such as a density fluctuation, a dust echo or a supernova bump (which could also be ruled out by amplitude and onset time). Other explanations involving an refreshed energy release such as a double jet (Berger et al. 2003) or a patchy shell (Meszaros et al. 1998) can not be discarded. This injection delays the break, that would be expected for about 1.6 days, to about 30 days and, mainly due to the effect of the equal arrival time surface, transforms it to a very smooth break that expands over a decade in time.

To explain the flattening seen in the earliest points of the light curve we have studied the case of a collimated fireball seen off-axis, as predicted by some unified models (Yamazaki et al. 2002) that simultaneously intend to explain LGRBs, XRR bursts and XRFs by only varying the viewing angle. Our fit accounts reasonably well for the multiband and long scale behaviour of the light curve. However, the fits obtained with an on-axis model with an additional early injection or a low initial gamma factor (dirty fireball) can also interpret the data (although returning worse fits) and can not be ruled out.

Regarding the energetics of the afterglow we find that, with an observed peak energy of $\sim 20 \mathrm{keV}$ and a fluence of $\sim 3.3 \times$ $10^{-6} \mathrm{erg} \mathrm{cm}^{-2}(2-400 \mathrm{keV})$ it has an isotropic equivalent energy release in $\gamma$-rays $E_{\gamma \text {,iso }} \gtrsim 1.3 \times 10^{52} \mathrm{erg}$, at least 6 times greater than the predicted by $E_{\text {peak }}-E_{\text {iso }}$ relation (Amati 2006).

We encourage polarimetric observations of XRR bursts and XRF events (i.e. Gorosabel et al. 2006) as they will be extremely useful to better understand the physics and geometry of the emission and to discriminate between energy models when explaining the fluctuations seen in the light curves.

Acknowledgements. We acknowledge the generous allocation of observing time by different Time Allocation Committees. This work was partially supported by the Spanish MCyT under programmes AYA2004-01515 and ESP2005-07714-C03-03 (including FEDER funds), RFBR grants 04-02-16300 and 05-02-17744 and grant NSh-784.2006.2. The Dark Cosmology Centre is supported by the Danish National Research Foundation. A.d.U.P. acknowledges support from FPU grant AP2002-0446 from the Spanish MCyT. G.J. acknowledges support from the Icelandic Research Council.

\section{References}

Allen, C. W. 2000, Astrophysical Quantities, 4th Ed. (London: Athlone) Amati, L. 2006, MNRAS, 372, 233

Berger, E., Kulkarni, S. R., Pooley, G., et al. 2003, Nature, 426, 154

Berger, E., Gladders, M., \& Oemler, G. 2005, GRB Coordinates Network, 3201

Castro-Tirado, A. J., Brandt, S., Lund, N., et al. 1994, Gamma-Ray Bursts, AIP Conf. Proc., 307, 17

Capalbi, M., Malesani, D., Perri, M., et al. 2006, A\&A, in press [arXiv: astro-ph/0610845]

Covino, et al. 2007, submitted

Chincarini, G., Moretti, A., Romano, P., et al. 2005 [arXiv: astro-ph/0506453]

Foley, R. J., Perley, D. A., Pooley, D., et al. 2006, ApJ, 645, 450

Fruchter, A. S., Levan, A. J., Strolger, L., et al. 2006, Nature, 441, 463 Fukugita, M., Shimasaku, K., \& Ichikawa, T. 1995, PASP, 107, 945 Gorosabel, J., Larionov, V., Castro-Tirado, A. J., et al. 2006, A\&A, 459, L33

Guilloteau, S., Delannoy, J., Downes, D., et al. 1992, A\&A, 262, 624 Granot, J., Ramirez-Ruiz, E., \& Perna, R. 2005, ApJ, 630, 1003

Huang, K. Y., Ip, W. H., Kinoshita, D., et al. 2005b, GRB Coordinates Network, 3196

Heise, J., in't Zand, J., Kippen, R. M., \& Woods, P. M. 2001, Gamma-ray Bursts in the Afterglow Era, 16

Jóhannesson, G., Björnsson, G., \& Gudmundsson, E. H. 2006, ApJ, 647, 1238

Kann, D. A., Klose, S., \& Zeh, A. 2006, ApJ, 641, 993

Kinney, A. L., Calzetti, D., Bohlin, R. C., et al. 1996, ApJ, 467, 38

Landolt, A. U. 1992, AJ, 104, 340

Meszaros, P., Rees, M. J., \& Wijers, R. A. M. J. 1998, ApJ, 499, 301

Nousek, J. A., Kouveliotou, C., Grupe, D., et al. 2006, ApJ, 642, 389

Pei, Y. C. 1992, ApJ, 395, 130

Prochaska, J. X., Bloom, J. S., Chen, H.-W., Foley, R. J., \& Roth, K. 2005, GRB Coordinates Network, 3204

Reichart, D. E. 2001, ApJ, 554, 643

Sakamoto, T., Ricker, G., Atteia, J.-L., et al. 2005a, GRB Coordinates Network, 3189

Sakamoto, T., Lamb, D. Q., Kawai, N., et al. 2005b, ApJ, 629, 311

Sari, R., Piran, T., \& Halpern, J. P. 1999, ApJ, 519, L17

Schlegel, D. J., Finkbeiner, D. P., \& Davis, M. 1998, ApJ, 500, 525

Soderberg, A. M. 2005a, GRB Coordinates Network, 3210

Soderberg, A. M. 2005b, GRB Coordinates Network, 3234

Stanek, K. Z., Garnavich, P. M., Nutzman, P. A., et al. 2005, ApJ, 626, L5

de Ugarte Postigo, A., Komorova, V., Fathkullin, T., et al. 2005a, GRB Coordinates Network, 3192

de Ugarte Postigo, A., et al. 2005b, JIBARO: Un conjunto de utilidades para la reducción y análisis automatizado de imágenes, in Astrofísica Robótica en España, ed. A. J. Castro-Tirado, B. A. de la Morena, \& J. Torres, Madrid, 35

de Ugarte Postigo, A., Castro-Tirado, A. J., Gorosabel, J., et al. 2005c, A\&A, 443,841

de Ugarte Postigo, A., Castro-Tirado, A. J., Guziy, S., et al. 2006, ApJ, 648, L83

Wells, A. A., Abbey, A. F., Osborne, J. P., et al. 2005, GRB Coordinates Network, 3191

Yamazaki, R., Ioka, K., \& Nakamura, T. 2002, ApJ, 571, L31

Yoshida, A., \& Murakami, T. 1994, Gamma-Ray Bursts, AIP Conf. Proc., 307, 333

Zhang, B., Fan, Y. Z., Dyks, J., et al. 2006, ApJ, 642, 354 
A. de Ugarte Postigo et al.: Extensive multiband study of the X-ray rich GRB 050408, Online Material p 1

\section{Online Material}


A. de Ugarte Postigo et al.: Extensive multiband study of the X-ray rich GRB 050408, Online Material p 2

\section{Appendix A: Calibration stars}

Table A.1. Calibration stars in the field of GRB 050408, as marked in Fig. A.1.

\begin{tabular}{rccccccc}
\hline \hline$\#$ & RA (J2000) & Dec (J2000) & $U$ & $B$ & $V$ & $R$ & $I$ \\
\hline 1 & $12: 02: 09.16$ & $+10: 49: 24.1$ & $19.114 \pm 0.053$ & $19.174 \pm 0.024$ & $18.601 \pm 0.009$ & $18.213 \pm 0.008$ & $17.835 \pm 0.024$ \\
2 & $12: 02: 10.38$ & $+10: 51: 37.3$ & $18.982 \pm 0.053$ & $19.241 \pm 0.024$ & $18.776 \pm 0.009$ & $18.438 \pm 0.008$ & $18.062 \pm 0.025$ \\
3 & $12: 02: 08.70$ & $+10: 51: 52.4$ & $19.743 \pm 0.054$ & $18.759 \pm 0.023$ & $17.167 \pm 0.008$ & $16.125 \pm 0.007$ & $14.776 \pm 0.022$ \\
4 & $12: 02: 14.68$ & $+10: 54.02 .8$ & $18.200 \pm 0.051$ & $18.508 \pm 0.023$ & $18.028 \pm 0.008$ & $17.671 \pm 0.007$ & $17.292 \pm 0.023$ \\
5 & $12: 02: 11.76$ & $+10: 54: 59.6$ & $18.204 \pm 0.051$ & $17.855 \pm 0.023$ & $17.089 \pm 0.008$ & $16.627 \pm 0.007$ & $16.218 \pm 0.022$ \\
6 & $12: 02: 22.95$ & $+10: 53: 57.8$ & $18.896 \pm 0.051$ & $19.098 \pm 0.023$ & $18.638 \pm 0.009$ & $18.300 \pm 0.008$ & $17.980 \pm 0.024$ \\
7 & $12: 02: 25.20$ & $+10: 51: 43.6$ & $20.034 \pm 0.058$ & $19.143 \pm 0.023$ & $17.681 \pm 0.008$ & $16.742 \pm 0.007$ & $15.695 \pm 0.022$ \\
8 & $12: 02: 24.70$ & $+10: 49: 54.7$ & $17.237 \pm 0.051$ & $17.406 \pm 0.023$ & $17.020 \pm 0.008$ & $16.652 \pm 0.007$ & $16.324 \pm 0.022$ \\
9 & $12: 02: 17.02$ & $+10: 51: 45.5$ & $20.510 \pm 0.064$ & $20.805 \pm 0.030$ & $20.216 \pm 0.018$ & $19.673 \pm 0.020$ & $18.951 \pm 0.041$ \\
10 & $12: 02: 14.76$ & $+10: 52: 54.6$ & - & $21.885 \pm 0.058$ & $20.528 \pm 0.019$ & $19.617 \pm 0.014$ & $18.631 \pm 0.028$ \\
11 & $12: 02: 17.10$ & $+10: 51: 02.5$ & - & $23.674 \pm 0.051$ & $22.376 \pm 0.098$ & $21.823 \pm 0.092$ & $20.947 \pm 0.137$ \\
12 & $12: 02: 11.32$ & $+10: 51: 11.3$ & $21.129 \pm 0.089$ & $21.249 \pm 0.034$ & $20.810 \pm 0.023$ & $20.507 \pm 0.029$ & $20.025 \pm 0.066$ \\
\hline
\end{tabular}


A. de Ugarte Postigo et al.: Extensive multiband study of the X-ray rich GRB 050408, Online Material p 3

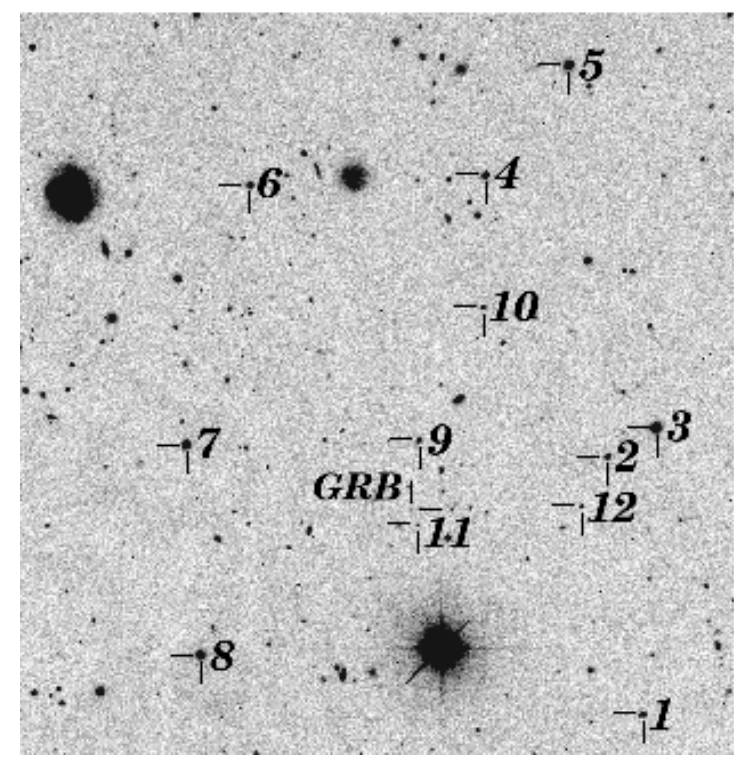

Fig. A.1. Identification chart of GRB 050408. The calibration stars and the afterglow location have been marked. The field of view is $6.0^{\prime} \times$ $6.5^{\prime}$, North is to the top and East to the left.

Optical photometric calibration is based on the observation of several standard fields (Landolt 1992) using the $1.54 \mathrm{~m}$ Danish at La Silla (Chile) and the $1.5 \mathrm{~m}$ telescope at Sierra Nevada Observatory (Spain). From these observations we derive 12 secondary standards of different brightnesses in the field of the GRB. Identification of these stars is shown in Fig. A.1, while their photometric values are displayed in Table A.1.

\section{Appendix B: Observations}

In the following tables we use the HETE-2 onset time $t_{0}=$ 2005 April 08 16:22:50.93 UT.
Table B.1. Observations in millimetre wavelengths from the Plateau du Bure interferometer (compact 6-antenna configuration on all dates). The errors are based on point-source fits in the UV plane to the phase center.

\begin{tabular}{cccc}
\hline \hline$\left(t-t_{0}\right)$ (days) & Band $(\mathrm{GHz})$ & Flux $(\mathrm{mJ})$ & $3-\sigma$ limit $(\mathrm{mJy})$ \\
\hline 3.23 & 86.789 & $0.0 \pm 0.3$ & 0.9 \\
3.23 & 229.068 & $1.0 \pm 1.6$ & 4.8 \\
5.19 & 115.477 & $0.5 \pm 0.8$ & 2.5 \\
5.19 & 232.295 & $3.3 \pm 1.8$ & 5.4 \\
10.34 & 86.251 & $0.9 \pm 0.3$ & $0.9^{a}$ \\
10.34 & 232.171 & $8.4 \pm 2.3$ & $6.9^{a}$ \\
11.19 & 108.995 & $-1.7 \pm 1.7$ & 5.2 \\
11.19 & 228.534 & $-9.9 \pm 6.5$ & 19.5 \\
12.40 & 108.995 & $-0.9 \pm 0.7$ & 2.2 \\
12.40 & 228.534 & $3.6 \pm 2.7$ & 8.2 \\
14.29 & 111.619 & $-0.8 \pm 0.4$ & 1.3 \\
14.29 & 224.680 & $1.4 \pm 2.1$ & 6.3 \\
\hline
\end{tabular}

${ }^{a}$ The faint detections found on this epoch are considered to be due to a statistical fluctuation or to an unusual event of interstellar scattering at high galactic latitud, taking into account the non detection the next night and the extreme spectral slope.

Table B.2. Observations in radio wavelengths from the Giant Metrewave Radio Telescope (GMRT) and Radio Astronomical Telescope Academy Nauk (RATAN-600).

\begin{tabular}{cccc}
\hline \hline$\left(t-t_{0}\right)$ (days) & Telescope & Band $(\mathrm{GHz})$ & 3- $\sigma$ limit $(\mathrm{mJy})$ \\
\hline 13.0 & GMRT & 1.28 & 0.45 \\
13.0 & RATAN-600 & 8.4 & 5.0 \\
\hline
\end{tabular}


A. de Ugarte Postigo et al.: Extensive multiband study of the X-ray rich GRB 050408, Online Material p 4

Table B.3. Optical observations carried out for GRB 050408. The magnitudes are in the Vega system and are not corrected for Galactic reddening.

\begin{tabular}{|c|c|c|c|c|c|}
\hline  & Tel. + Inst. & Filter & $\overline{\overline{T \exp (\mathrm{s})}}$ & $\overline{\mathrm{Mag}}$ & $\overline{\overline{\text { ErMag }}}$ \\
\hline 0.4780 & Dk1.54 m+DFOSC & $\bar{U}$ & 9000 & 23.09 & 0.18 \\
\hline 1.5939 & $4.0 \mathrm{mKPNO}$ & $U$ & 1600 & 23.75 & 0.26 \\
\hline 0.1297 & $6.0 \mathrm{mBTA}+\mathrm{SCORPIO}$ & $\bar{B}$ & 600 & 22.37 & 0.05 \\
\hline 0.1385 & $6.0 \mathrm{mBTA}+\mathrm{SCORPIO}$ & $B$ & 500 & 22.44 & 0.05 \\
\hline 0.1467 & $6.0 \mathrm{mBTA}+\mathrm{SCORPIO}$ & $B$ & 600 & 22.46 & 0.05 \\
\hline 0.1554 & $6.0 \mathrm{mBTA}+\mathrm{SCORPIO}$ & $B$ & 600 & 22.55 & 0.05 \\
\hline 0.2301 & $6.0 \mathrm{mBTA}+\mathrm{SCORPIO}$ & $B$ & 500 & 22.79 & 0.07 \\
\hline 0.3712 & Dk1.54 m+DFOSC & $B$ & 1200 & 23.05 & 0.10 \\
\hline 0.5681 & Dk1.54 m+DFOSC & $B$ & 1200 & 23.54 & 0.17 \\
\hline 1.5001 & Dk1.54 m+DFOSC & $B$ & 9000 & 24.26 & 0.10 \\
\hline 242.5213 & 3.5 mCAHA & $B$ & 1800 & $25.32^{4}$ & 0.22 \\
\hline 0.2245 & $6.0 \mathrm{mBTA}+\mathrm{SCORPIO}$ & $V$ & 300 & 22.11 & 0.06 \\
\hline 0.3599 & Dk1.54 m+DFOSC & $V$ & 600 & 22.24 & 0.09 \\
\hline 1.6206 & $4.0 \mathrm{mKPNO}$ & $V$ & 1200 & 23.98 & 0.22 \\
\hline 2.4394 & $4.0 \mathrm{mCTIO}$ & $V$ & 1500 & 24.10 & 0.17 \\
\hline 0.0155 & Kiso 1.05 Schmidt & $R \mathrm{c}$ & 300 & $>19.50$ & - \\
\hline 0.0388 & $1.0 \mathrm{mLOT}$ & $R \mathrm{c}^{5}$ & 180 & 20.34 & 0.10 \\
\hline 0.0859 & $1.0 \mathrm{mZeiss}$ & $R \mathrm{c}$ & 900 & 20.99 & 0.09 \\
\hline 0.0919 & $1.0 \mathrm{mLOT}$ & $R \mathrm{c}^{5}$ & 180 & 20.80 & 0.18 \\
\hline 0.0956 & $1.0 \mathrm{mLOT}$ & $R \mathrm{c}^{5}$ & 300 & 20.98 & 0.14 \\
\hline 0.1029 & $6.0 \mathrm{mBTA}+\mathrm{SCORPIO}$ & $R \mathrm{c}$ & 180 & 20.92 & 0.03 \\
\hline 0.1068 & $6.0 \mathrm{mBTA}+\mathrm{SCORPIO}$ & $R \mathrm{c}$ & 180 & 20.94 & 0.03 \\
\hline 0.1104 & $6.0 \mathrm{mBTA}+\mathrm{SCORPIO}$ & $R \mathrm{c}$ & 180 & 20.92 & 0.03 \\
\hline 0.1139 & $6.0 \mathrm{mBTA}+\mathrm{SCORPIO}$ & $R \mathrm{c}$ & 180 & 20.92 & 0.02 \\
\hline 0.1172 & $6.0 \mathrm{mBTA}+\mathrm{SCORPIO}$ & $R \mathrm{c}$ & 180 & 20.95 & 0.02 \\
\hline 0.1207 & $6.0 \mathrm{mBTA}+\mathrm{SCORPIO}$ & $R \mathrm{c}$ & 180 & 21.03 & 0.03 \\
\hline 0.1240 & $6.0 \mathrm{mBTA}+\mathrm{SCORPIO}$ & $R \mathrm{c}$ & 180 & 21.05 & 0.03 \\
\hline 0.1471 & RTT150+TFOSC & $R \mathrm{c}$ & 450 & 21.13 & 0.08 \\
\hline 0.1629 & RTT150+TFOSC & $R \mathrm{c}$ & 450 & 21.05 & 0.06 \\
\hline 0.1771 & RTT150+TFOSC & $R \mathrm{c}$ & 540 & 21.10 & 0.06 \\
\hline 0.1863 & RTT150+TFOSC & $R \mathrm{c}$ & 540 & 21.19 & 0.06 \\
\hline 0.1984 & RTT150+TFOSC & $R \mathrm{c}$ & 480 & 21.42 & 0.08 \\
\hline
\end{tabular}


A. de Ugarte Postigo et al.: Extensive multiband study of the X-ray rich GRB 050408, Online Material p 5

Table B.3. continued.

\begin{tabular}{|c|c|c|c|c|c|}
\hline$\left(t-t_{0}\right)$ (days) & Tel. + Inst. & Filter & $T \exp (\mathrm{s})$ & Mag & ErMag \\
\hline 0.2080 & RTT150+TFOSC & $R \mathrm{c}$ & 480 & 21.40 & 0.08 \\
\hline 0.2175 & RTT150+TFOSC & $R \mathrm{c}$ & 480 & 21.39 & 0.09 \\
\hline 0.2204 & 6.0 mBTA+SCORPIO & $R \mathrm{c}$ & 180 & 21.32 & 0.04 \\
\hline 0.2296 & RTT150+TFOSC & $R \mathrm{c}$ & 480 & 21.19 & 0.06 \\
\hline 0.2380 & RTT150+TFOSC & $R \mathrm{c}$ & 480 & 21.52 & 0.09 \\
\hline 0.2463 & RTT150+TFOSC & $R \mathrm{c}$ & 480 & 21.56 & 0.08 \\
\hline 0.2742 & RTT150+TFOSC & $R \mathrm{c}$ & 720 & 21.52 & 0.06 \\
\hline 0.2867 & RTT150+TFOSC & $R \mathrm{c}$ & 720 & 21.58 & 0.06 \\
\hline 0.3392 & RTT150+TFOSC & $R \mathrm{c}$ & 600 & 21.80 & 0.12 \\
\hline 0.3496 & RTT150+TFOSC & $R \mathrm{c}$ & 600 & 21.50 & 0.09 \\
\hline 0.3540 & Dk1.54 m+DFOSC & $R \mathrm{c}$ & 300 & 21.81 & 0.13 \\
\hline 0.3600 & RTT150+TFOSC & $R \mathrm{c}$ & 600 & 21.84 & 0.14 \\
\hline 0.3705 & RTT150+TFOSC & $R \mathrm{c}$ & 600 & 21.95 & 0.15 \\
\hline 0.4362 & Dk1.54 m+DFOSC & $R \mathrm{c}$ & 600 & 21.91 & 0.08 \\
\hline 0.5366 & Dk1.54 m+DFOSC & $R \mathrm{c}$ & 900 & 22.00 & 0.07 \\
\hline 0.5963 & Dk1.54 m+DFOSC & $R \mathrm{c}$ & 1200 & 22.02 & 0.08 \\
\hline 0.7166 & $2.0 \mathrm{mFTN}$ & $R \mathrm{c}$ & 2400 & 22.50 & 0.11 \\
\hline 1.4024 & Dk1.54 m+DFOSC & $R \mathrm{c}$ & 5700 & 23.08 & 0.09 \\
\hline 1.5864 & Dk1.54 m+DFOSC & $R \mathrm{c}$ & 3600 & 23.31 & 0.14 \\
\hline 2.4911 & Dk1.54 m+DFOSC & $R \mathrm{c}$ & 14700 & 23.86 & 0.17 \\
\hline 3.4506 & Dk1.54 m+DFOSC & $R \mathrm{c}$ & 14000 & 23.85 & 0.13 \\
\hline 5.1575 & RTT150+ANDOR CCD & $R \mathrm{c}$ & 9000 & 23.70 & 0.20 \\
\hline 5.5124 & Dk1.54 m+DFOSC & $R \mathrm{c}$ & 7200 & 23.72 & 0.18 \\
\hline 7.4663 & Dk1.54 m+DFOSC & $R \mathrm{c}$ & 15600 & 23.97 & 0.11 \\
\hline 30.2567 & $3.5 \mathrm{mTNG}$ & $R \mathrm{c}$ & 3600 & 24.64 & 0.17 \\
\hline 242.4885 & $3.5 \mathrm{mCAHA}$ & $R \mathrm{c}$ & 2500 & $24.60^{4}$ & 0.15 \\
\hline 0.3871 & Dk1.54 m+DFOSC & Ic & 1200 & 21.25 & 0.07 \\
\hline 0.6137 & Dk1.54 m+DFOSC & Ic & 1500 & 22.52 & 0.11 \\
\hline 6.4766 & Dk1.54 m+DFOSC & Ic & 13800 & 23.50 & 0.18 \\
\hline 242.5446 & $3.5 \mathrm{mCAHA}$ & Ic & 1500 & $>24.0$ & - \\
\hline-0.00340 & WIDGET & Unfiltered & 95 & $>9.7$ & - \\
\hline-0.00224 & WIDGET & Unfiltered & 95 & $>9.7$ & - \\
\hline-0.00108 & WIDGET & Unfiltered & 95 & $>9.7$ & - \\
\hline 0.00007 & WIDGET & Unfiltered & 95 & $>9.8$ & - \\
\hline 0.00123 & WIDGET & Unfiltered & 95 & $>9.7$ & - \\
\hline
\end{tabular}

${ }^{4}$ Host galaxy magnitude.

${ }^{5} V R$ broad band filter was transformed to $R \mathrm{c}$. 